\title{
BMJ Open Normal range of serum potassium, prevalence of dyskalaemia and associated factors in Chinese older adults: a cross-sectional study
}

Aoming Jin (1) , ${ }^{1}$ Minghui Zhao, ${ }^{2}$ Yihong Sun, ${ }^{3}$ Xiangxian Feng, ${ }^{4}$ Ruijuan Zhang, ${ }^{5}$ Qianku Qiao, ${ }^{6}$ Hongxia Wang, ${ }^{7}$ Jianhui Yuan, ${ }^{4}$ Yuqi Wang, ${ }^{5}$ Lili Cheng, ${ }^{6}$ Hui Zhang, ${ }^{7}$ Hui-Juan $\mathrm{Li}^{1}{ }^{1}$ Yangfeng $\mathrm{Wu}^{1,8}$

To cite: Jin A, Zhao M, Sun Y, et al. Normal range of serum potassium, prevalence of dyskalaemia and associated factors in Chinese older adults: a cross-sectional study. BMJ Open 2020;10:e039472. doi:10.1136/ bmjopen-2020-039472

- Prepublication history and additional material for this paper are available online. To view these files, please visit the journal online (http://dx.doi org/10.1136/bmjopen-2020039472).

Received 16 April 2020 Revised 23 July 2020

Accepted 14 September 2020

Check for updates

(C) Author(s) (or their employer(s)) 2020. Re-use permitted under CC BY-NC. No commercial re-use. See rights and permissions. Published by BMJ.

For numbered affiliations see end of article.

Correspondence to Dr Yangfeng Wu; wuyf@bjmu.edu.cn

\section{ABSTRACT}

Objective To investigate the normal range of serum potassium, the prevalence of dyskalaemia and the associated factors in Chinese older adults.

Design A cross-sectional study conducted from

September 2017 to March 2018.

Setting Forty-eight community elderly care facilities in four regions in northern China.

Participants A total of 1266 (308 apparently healthy and 958 unhealthy) participants 55 years or older and with fasting serum potassium measured.

Main outcome measures and methods Serum potassium $<3.5 \mathrm{mEq} / \mathrm{L}$ and $>5.5 \mathrm{mEq} / \mathrm{L}$ (guidelines definition) and $<2.5$ th and $>97.5$ th percentiles of the distribution among healthy participants (our study definition) were both used to define hypokalaemia and hyperkalaemia, respectively. Multivariable generalised estimating equation models were used to adjust for clustering effect in the analyses of factors associated with risk of dyskalaemia and with variations in serum potassium.

Results The study participants had a mean age of 70 (8.8) years. Among apparently healthy participants, the 2.5 th and 97.5th percentiles of serum potassium distribution were $3.7 \mathrm{mEq} / \mathrm{L}$ and $5.3 \mathrm{mEq} / \mathrm{L}$, respectively. Using the study definition, the prevalence of hyperkalaemia was $4.3 \%$ (95\% $\mathrm{Cl} 3.2 \%$ to $5.4 \%)$ and of hypokalaemia was $4.0 \%(95 \% \mathrm{Cl}$ $2.9 \%$ to $5.1 \%$ ). Multivariable analyses showed that risk of hyperkalaemia was associated with unhealthy conditions $(\mathrm{OR}=2.21 ; 95 \% \mathrm{Cl} 1.17$ to 4.18$)$; risk of hypokalaemia was associated with unhealthy conditions ( $\mathrm{OR}=2.56 ; 95 \% \mathrm{Cl} 1.05$ to 6.23$)$, older age $(\mathrm{OR}=1.70$ per 10-year increase; $95 \% \mathrm{Cl}$ 1.04 to 2.79 ) and region ( $\mathrm{OR}=16.87 ; 95 \% \mathrm{Cl} 6.41$ to 44.38 ); and higher serum potassium was associated with male gender (mean difference $(\mathrm{MD})=0.12 ; 95 \% \mathrm{Cl} 0.05$ to 0.19 ) and estimated glomerular filtration rate $<60 \mathrm{~mL} / \mathrm{min} / 1.73 \mathrm{~m}^{2}$ ( $\mathrm{MD}=0.29 ; 95 \% \mathrm{Cl} 0.12$ to 0.46 ). Using the guidelines definition, hyperkalaemia accounted for $2.7 \%(1.8 \%, 3.6 \%)$ and hypokalaemia $1.8 \%(1.1 \%, 2.5 \%)$. Analyses of the associated factors showed similar trends.

Conclusions The study suggested a narrower normal range of serum potassium for defining dyskalaemia, which was common in older Chinese and more prevalent in unhealthy ones.

Trial registration number NCT03290716; Pre-results.
Strengths and limitations of this study

- A large number of apparently healthy study participants provided data for defining the normal range of serum potassium.

Dyskalaemia was defined using both clinical guidelines and study-based definitions.

- The prevalence of dyskalaemia was reported for the first time among Chinese older adults.

- Dyskalaemia was defined merely on a single blood test of serum potassium.

- The study population was institutionalised and predominantly composed of men.

\section{INTRODUCTION}

Dyskalaemia, either hyperkalaemia or hypokalaemia, may lead to muscular weakness and cardiac arrhythmias and is associated with increased hospitalisation and mortality. ${ }^{1-3}$ Besides its life-threatening influences, patients with dyskalaemia might unduly discontinue administration of certain medications that bear benefits on clinical outcomes, such as ACE inhibitors (ACEI) and diuretics. ${ }^{45}$ It is clinically important to characterise the epidemiology of dyskalaemia in the general population.

Existing studies that reported on the prevalence of dyskalaemia were primarily conducted in patients with illnesses such as renal insufficiency, heart failure and diabetes mellitus, or in the setting of emergency departments, including some with data from healthcare claims of a broader range of patient population. ${ }^{67}$ Only few studies were conducted in the general population and all were done three decades ago and all in Western populations. ${ }^{8-10}$ Further, there is not universally agreed reference ranges that are used to diagnose dyskalaemia, particularly 
to diagnose hyperkalaemia, and references were mostly based on studies in Western populations.

Many factors have been reported to be associated with risk of dyskalaemia. ${ }^{311-13}$ Similar to data on the prevalence, current evidence on risk factors for dyskalaemia was mainly from studies among patients. There is a lack of data from unhospitalised populations. Thus, the present study aims to preliminarily understand (1) the distribution and normal range of serum potassium, (2) the prevalence of hyperkalaemia and hypokalaemia, and (3) the factors associated with risk of hyperkalaemia and hypokalaemia and with variations in serum potassium level, among Chinese older adults.

\section{METHODS}

\section{Study population}

The DECIDE-Salt study is an ongoing cluster randomised controlled trial with two-by-two factorial design to evaluate the effect of two salt reduction strategies, stepwise salt supply control and salt substitute, in lowering blood pressure. The details of the DECIDE-Salt trial have been presented elsewhere. ${ }^{14}$ Briefly, 48 community elderly care facilities from four regions, two in rural areas (Changzhi and Yangcheng in Shanxi Province) and two in urban areas (Xi'an in Shaanxi Province and Hohhot in Inner Mongolia Autonomous Region), were recruited. The type of facilities in China varies according to the services provided, and these mainly include assisted living homes in rural areas and nursing homes in urban areas. Assisted living homes provide meals, accommodations and recreational activities for older adults otherwise living alone to provide for their basic needs. Nursing homes provide basic medical care in addition to assisted living. To be adopted by an elderly care facility, candidates should provide health check-up results showing no communicable diseases and no acute medical conditions that require hospitalisation. ${ }^{15}$ All facilities included in the study were randomly assigned into one of four groups: stepwise salt supply control and salt substitute, stepwise salt supply control only, salt substitute only, and neither. The interventions last for 2 years. Written informed consent was obtained from every participant.

For research purposes, we used data from the baseline investigation of DECIDE-Salt trial, which was conducted from September 2017 to March 2018. To be included in the trial, participants should be 55 years and older, are expected to live in the facility for at least 2 years and have signed informed consent. Unstable residents (expected absence from the facility more than 1 month per year) and those with incomplete blood pressure measurements at baseline were excluded from the trial. Among all 1606 participants in the DECIDE-Salt trial, 340 refused drawing of blood sample at baseline and hence were excluded from the present study. Differences in baseline characteristics between included and excluded participants are given in online supplemental table 1.

\section{Study measurements and methods}

We used an interviewer-administered questionnaire to collect data on demographics, lifestyle behaviours, history of diseases and medication use. For antihypertension medications, the generic names were recorded by checking the labels on the containers. Blood pressure was measured on the right arm three times with an interval of at least $30 \mathrm{~s}$, using standard methods. ${ }^{16}$ We used the mean of three blood pressure measurements for analysis. Overnight fasting blood samples were drawn by a nurse and were shipped to the central laboratory in Beijing for biochemistry assays. ${ }^{17}$ Serum creatinine was measured using a Roche enzymatic assay on a Roche Cobas c501 platform with the CREP2-Creatinine Plus V.2 reagent. Serum potassium was measured with the ion-selective electrode method using a Roche Cobas c501 platform.

\section{Definitions}

According to the current guidelines, ${ }^{18}{ }^{19}$ hyperkalaemia is defined as serum potassium $>5.5 \mathrm{mEq} / \mathrm{L}$ (to convert to $\mathrm{mmol} / \mathrm{L}$, multiply the value by 1.0 ), while hypokalaemia is defined as serum potassium $<3.5 \mathrm{mEq} / \mathrm{L}$ (referred to as the 'guidelines definition'). We also defined hyperkalaemia as serum potassium $>97.5$ th percentile and hypokalaemia as serum potassium $<2.5$ th percentile of the distribution in the healthy participants in our study (referred to as the 'study definition'). Hypertension was defined as systolic blood pressure $\geq 140 \mathrm{~mm} \mathrm{Hg}$ or diastolic blood pressure $\geq 90 \mathrm{~mm}$ $\mathrm{Hg}$, or on antihypertension medications within 2 weeks. ${ }^{16}$ Diabetes mellitus was defined as fasting glucose $\geq 126 \mathrm{mg}$ / dL $(7.0 \mathrm{mmol} / \mathrm{L})$ or by self-reported history of diabetes. Stroke, coronary heart disease, cancer, chronic obstructive pulmonary disease and chronic kidney disease were defined according to participants' self-reported information. Antihypertension medications were classified as potassium-sparing medications including ACEI/angiotensin receptor blocker (ARB), $\beta$-blocker and potassium-sparing diuretics; kaliuretic diuretics; and other medications including calcium channel blocker, $\alpha$-blocker and so on. Estimated glomerular filtration rate (eGFR) was calculated with the Chronic Kidney Disease Epidemiology Collaboration (CKD-EPI) creatinine equation using serum creatinine. ${ }^{20}$ Unhealthy conditions included hypertension, diabetes mellitus, coronary heart disease, stroke, chronic kidney disease, cancer, chronic obstructive pulmonary disease or being bedridden. Apparently healthy condition was defined as having none of the above unhealthy conditions. In addition, dementia and other severe mental conditions had been excluded from the DECIDE-Salt trial. Government regulations precluded any person with communicable diseases or acute medical conditions that require hospitalisation to be adopted to the facilities. For the population used to analyse the normal range of serum potassium, we further excluded participants with $\mathrm{eGFR}<60 \mathrm{~mL} / \mathrm{min} / 1.73 \mathrm{~m}^{2}$.

\section{Statistical methods}

We described the mean serum potassium and the prevalence of dyskalaemia by participants' health condition as well as 
region, gender and age groups. We used analysis of variance and Pearson $\chi^{2}$ test to compare differences between groups. The 2.5th and 97.5th percentiles of distribution among apparently healthy participants were used to define the normal range of serum potassium. For risk of dyskalaemia, the analysis was done separately for hyperkalaemia and hypokalaemia. Associated factors considered included age, gender, region, health condition, antihypertension medication use and eGFR. A generalised estimating equation (GEE) model with an exchangeable covariance structure was employed to confirm factors independently associated with risk of dyskalaemia and variations in serum potassium, adjusting for the clustering effect at facility level. To increase statistical power and reduce collinearity, we combined diabetes and coronary heart disease in our multivariable models since they showed the same trends in association with the outcomes in the univariate analyses. There were 60 participants with missing data on antihypertension medication use, and we imputed these missing data with multiple imputation by fully conditional specification approach. ${ }^{21}$ All statistical tests were two-sided and a p value less than 0.05 was considered statistically significant. All statistical analyses were carried out using SAS V.9.4.

\section{Patient and public involvement}

Patients and/or the public were not involved in the design or conduct of this study.

\section{RESULTS}

\section{Characteristics of study population}

The study population of 1266 participants had a mean age of 70 years, ranging from 55 to 102 years old. Over $80 \%$ were men. Majority (64\%) had hypertension but nearly $40 \%$ of them were not taking any antihypertension medication (table 1). Among those on medication, 34\% were taking kaliuretic and $18 \%$ potassium-sparing medications. Overall, $958(76 \%)$ participants were defined unhealthy.

\section{Distribution of serum potassium levels}

The distribution of serum potassium levels in the study population is shown in figure 1 . The mean serum potassium among all, apparently healthy and unhealthy participants was $4.4(0.5) \mathrm{mEq} / \mathrm{L}, 4.4(0.4) \mathrm{mEq} / \mathrm{L}$ and 4.5 $(0.5) \mathrm{mEq} / \mathrm{L}$, respectively. The distribution in apparently healthy participants was close to symmetric, with the median at $4.4 \mathrm{mEq} / \mathrm{L}$ and the 2.5th and 97.5th percentiles at $3.7 \mathrm{mEq} / \mathrm{L}$ and $5.3 \mathrm{mEq} / \mathrm{L}$, respectively.

The mean serum potassium by age, sex and region is given in online supplemental table 2.

\section{Prevalence of dyskalaemia}

Using the guidelines definition, the prevalence of hyperkalaemia was $2.7 \%$ in all and differed significantly between apparently healthy and unhealthy participants $(1.0 \%$ vs $3.2 \%, \mathrm{p}=0.03)$. The prevalence of hypokalaemia was $1.8 \%$ in all participants, and the difference in the prevalence between healthy $(0.6 \%)$ and unhealthy $(2.2 \%)$ participants was borderline significant $(\mathrm{p}=0.08)$.

Using the 2.5 th and 97.5 th percentiles in apparently healthy participants to define hyperkalaemia (serum potassium $>5.3 \mathrm{mEq} / \mathrm{L}$ ) and hypokalaemia (serum potassium $<3.7 \mathrm{mEq} / \mathrm{L}$ ), the prevalence in unhealthy participants would be $5.0 \%$ for hyperkalaemia and $4.5 \%$ for hypokalaemia, respectively, and the prevalence among all participants would be $4.3 \%$ and $4.0 \%$, respectively. Comparisons between healthy and unhealthy participants showed a significant difference in the prevalence of hyperkalaemia $(\mathrm{p}=0.04)$ and $\mathrm{a}$

\begin{tabular}{|c|c|}
\hline Variables & Statistics \\
\hline Number of participants & 1266 \\
\hline Age (years), mean (SD) & $69.8(8.8)$ \\
\hline Men, n (\%) & $1048(82.8)$ \\
\hline \multicolumn{2}{|l|}{ Region, n (\%) } \\
\hline Changzhi & $472(37.3)$ \\
\hline Yangcheng & $285(22.5)$ \\
\hline Xi'an & $299(23.6)$ \\
\hline Hohhot & $210(16.6)$ \\
\hline Current smoking, n (\%) & $488(38.6)$ \\
\hline Current drinking, $\mathrm{n}(\%)$ & $134(10.6)$ \\
\hline \multicolumn{2}{|l|}{ Unhealthy conditions, n (\%) } \\
\hline Hypertension & $815(64.4)$ \\
\hline Stroke & $338(26.7)$ \\
\hline Diabetes mellitus & $149(11.8)$ \\
\hline Coronary heart disease & $128(10.1)$ \\
\hline Chronic kidney disease & $30(2.4)$ \\
\hline $\begin{array}{l}\text { Chronic obstructive pulmonary } \\
\text { disease }\end{array}$ & $11(0.9)$ \\
\hline Cancer & $8(0.6)$ \\
\hline Bedridden & $66(5.2)$ \\
\hline \multicolumn{2}{|l|}{ Antihypertension medication use, n (\%) } \\
\hline Potassium-sparing medications ${ }^{*} \dagger$ & $119(9.9)$ \\
\hline Kaliuretic diuretics ${ }^{\star}$ & $161(13.4)$ \\
\hline Other antihypertension medications* $\neq$ & $260(21.6)$ \\
\hline \multicolumn{2}{|l|}{ eGFR, mL/min/1.73 m², n (\%) } \\
\hline$\geq 90$ & 707 (55.9) \\
\hline $60-89$ & $504(39.8)$ \\
\hline$<60$ & $55(4.3)$ \\
\hline
\end{tabular}

*Number of participants with missing data: 60 . †Include ACEI/ARB, $\beta$-blocker and potassium-sparing diuretics.

łlnclude calcium channel blocker, $\alpha$-blocker and so on. ACEI, ACE inhibitor; ARB, angiotensin receptor blocker; eGFR, estimated glomerular filtration rate. 

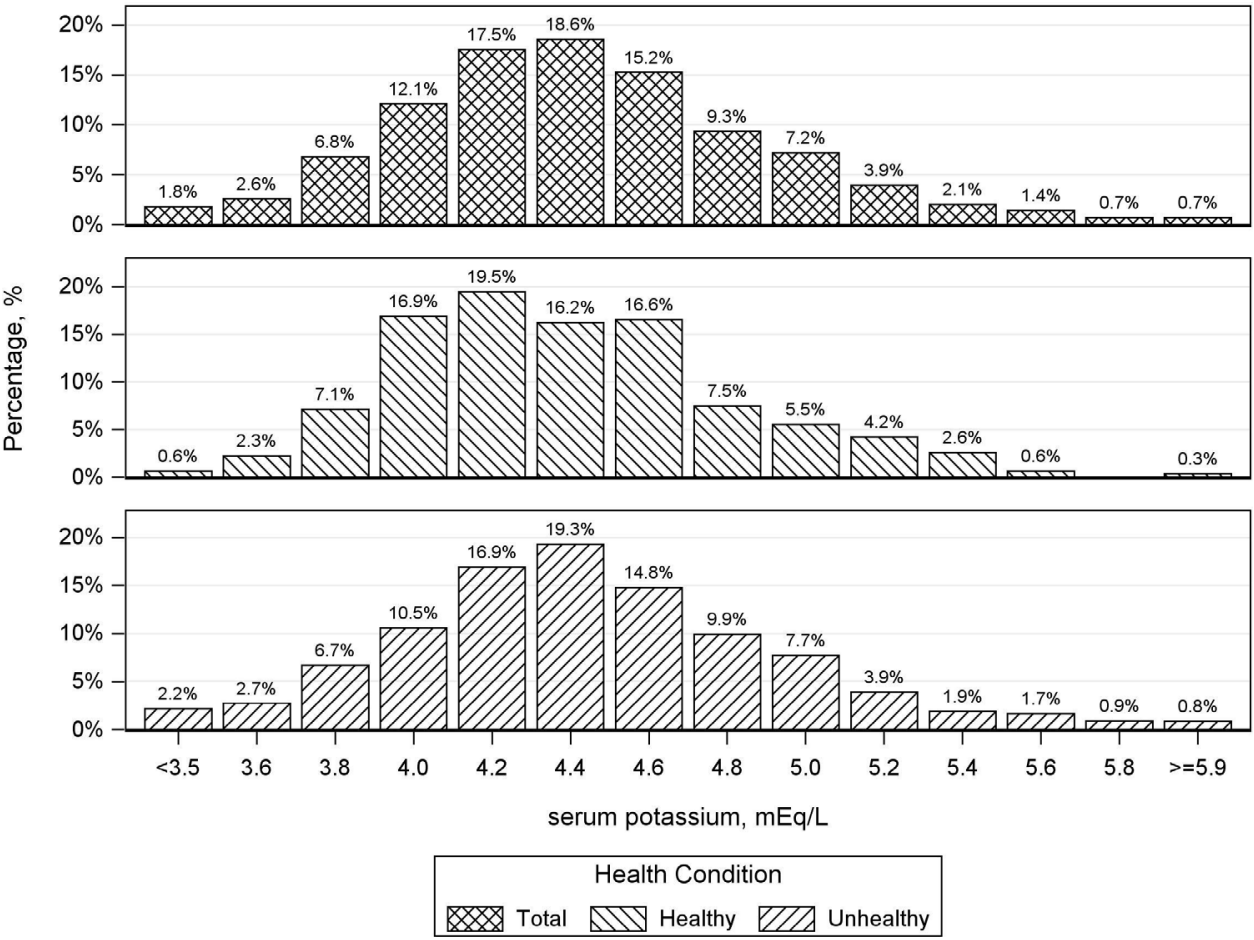

Figure 1 Distribution of serum potassium in all, apparently healthy and unhealthy participants. Unhealthy conditions included hypertension, diabetes mellitus, coronary heart disease, stroke, chronic kidney disease, cancer, chronic obstructive pulmonary disease or being bedridden.

borderline significant difference in the prevalence of hypokalaemia $(\mathrm{p}=0.08)$.

The prevalence of potassium abnormalities by age, sex and region using the guidelines definition and the study definition is given in online supplemental tables 3 to 6 .

\section{Factors associated with hyperkalaemia, hypokalaemia and serum potassium}

Using either the guidelines or our study definition, univariate analyses showed similar results. A higher risk of hyperkalaemia was significantly associated with unhealthy conditions, specifically hypertension and diabetes; a higher risk of hypokalaemia was significantly associated with region and unhealthy conditions, specifically coronary heart disease (guidelines definition only); and a higher level of serum potassium was associated with region and low eGFR $\left(<60 \mathrm{~mL} / \mathrm{min} / 1.73 \mathrm{~m}^{2}\right)$ (table 2$)$.

The GEE analyses adjusting for the clustering effect and covariables showed that a higher risk of hyperkalaemia was significantly associated with unhealthy conditions and region; a higher risk of hypokalaemia was significantly associated with unhealthy conditions, older age and region (our study definition); and a higher level of serum potassium was associated with low eGFR and male gender (model 1 in table 3 ).

To further understand the role of specific diseases and medications, we fit the GEE models with the specific unhealthy conditions replacing the overall health condition (model 2 in table 3 ). The results showed that a higher risk of hyperkalaemia was significantly associated with diabetes mellitus and/or coronary heart disease and low
eGFR, but not with stroke and other unhealthy conditions or any studied medication. No specific unhealthy conditions or medications were identified to be significantly associated with risk of hypokalaemia. Use of diuretics and antihypertension medications not affecting potassium homeostasis and having diseases other than hypertension, stroke, diabetes and/or coronary heart disease were associated with a lower level but having stroke was associated with a higher level of serum potassium.

\section{DISCUSSION}

\section{Normal range of serum potassium}

Our analysis on the distribution of serum potassium level among apparently healthy older adults indicated a normal range between $3.7 \mathrm{mEq} / \mathrm{L}$ and $5.3 \mathrm{mEq} / \mathrm{L}$, accounting for $95 \%$ of the healthy population. It was narrower than the current widely adopted normal range of 3.5-5.5 $\mathrm{mEq} / \mathrm{L} \cdot{ }^{1819}$ A narrower normal range of $3.6-5.2 \mathrm{mEq} / \mathrm{L}$ was reported previously in a study of 4524 healthy Chinese adults between 20 and 79 years old. ${ }^{22}$ Considering the associations of serum potassium with the risk of total death, cardiovascular death and end-stage renal disease were ' $U$ '-shaped, ${ }^{23}$ we believe a narrower normal range should better be defined and applied in clinical practice.

\section{Prevalence of dyskalaemia}

Our study revealed that, using either the guidelines definition $(4.5 \%)$ or our study definition $(8.3 \%)$, dyskalaemia was common among Chinese older adults living in elderly care facilities. 


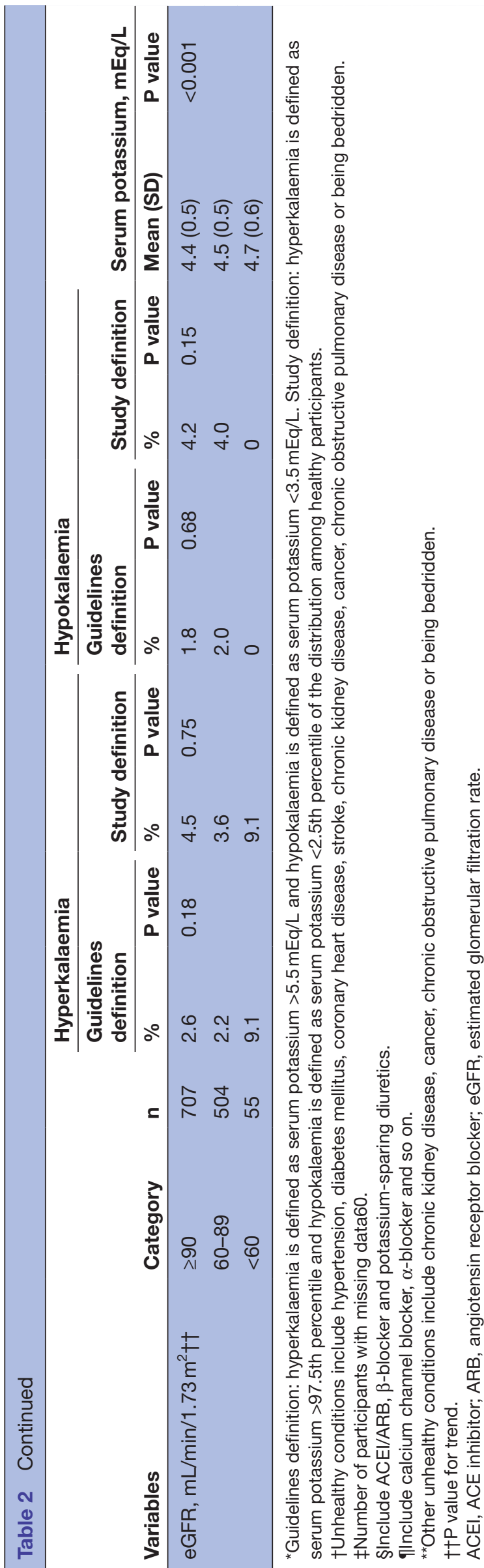

There are very few studies that reported the prevalence of dyskalaemia in the general population and none in a healthy population. The Rotterdam study among 5179 inhabitants with a mean age of 70.3 years reported that $0.9 \%$ had serum potassium $>5.3 \mathrm{mEq} / \mathrm{L}$ and $7.2 \%$ had serum potassium $<3.5 \mathrm{mEq} / \mathrm{L} .{ }^{9}$ In 1558 elderly individuals with a mean age of 81.4 years in an urban area of Stockholm, 2.8\% had serum potassium $>5.0 \mathrm{mEq} / \mathrm{L}$ and $2.5 \%$ had serum potassium $<3.5 \mathrm{mEq} / \mathrm{L} .{ }^{10}$ Another study in the USA among 15792 individuals aged 44-66 years reported that $2.1 \%$ of participants had serum potassium $\geq 5.5 \mathrm{mEq} / \mathrm{L}$ and $2.7 \%$ had serum potassium $<3.5 \mathrm{mEq} / \mathrm{L}$. These findings were generally comparable with our results.

\section{Associated factors}

To better understand the factors that may drive the development of dyskalaemia and the underlying mechanisms, we fit the study variables simultaneously with risk of hyperkalaemia, risk of hypokalaemia and variations in serum potassium level. The study variables chosen were based on the results from the univariate analyses and knowledge from previous studies. ${ }^{3711}$ Putting together the resulting associations with hyperkalaemia, hypokalaemia and serum potassium, the pattern of the associations differed from factor to factor, indicating the underlying mechanisms may differ very much.

First, low eGFR (below $60 \mathrm{~mL} / \mathrm{min} / 1.73 \mathrm{~m}^{2}$ ) was associated with a higher mean of serum potassium but not in a linear pattern. The association has been known for many years and the results were consistent throughout the studies. ${ }^{72}$ It is widely believed that renal insufficiency, resulting mostly from renal diseases, would reduce the excretion of potassium and hence increase serum potassium level, which finally leads to the development of hyperkalaemia. Our findings strongly support this hypothesis.

Unlike low eGFR, diabetes mellitus and/or coronary heart disease were associated with higher risk of both hyperkalaemia and hypokalaemia, but not with variations in serum potassium. These are also in line with previous findings. ${ }^{725} 26$ The explanations may be attributed to use of potassium homeostasis-affecting medications, polypharmacy treatment, disease pathology and multiple comorbidities. ${ }^{27} 28$ However, the role of medication use, drug-drug interactions and disease pathology can be hardly differentiated in this situation.

Among previously reported medications that affect potassium homeostasis, ${ }^{12}{ }^{13}$ spironolactone and ACEI/ ARB have been proven in randomised trials to increase serum potassium and the risk of hyperkalaemia, while kaliuretic medications reduce serum potassium and increase the risk of hypokalaemia. ${ }^{29}{ }^{30}$ In our study, use of kaliuretic medications was significantly associated with lower mean serum potassium and insignificantly associated with a higher risk of hypokalaemia. Use of potassium-sparing medications was not associated with serum potassium nor risk of hyperkalaemia. The reasons might largely be due 


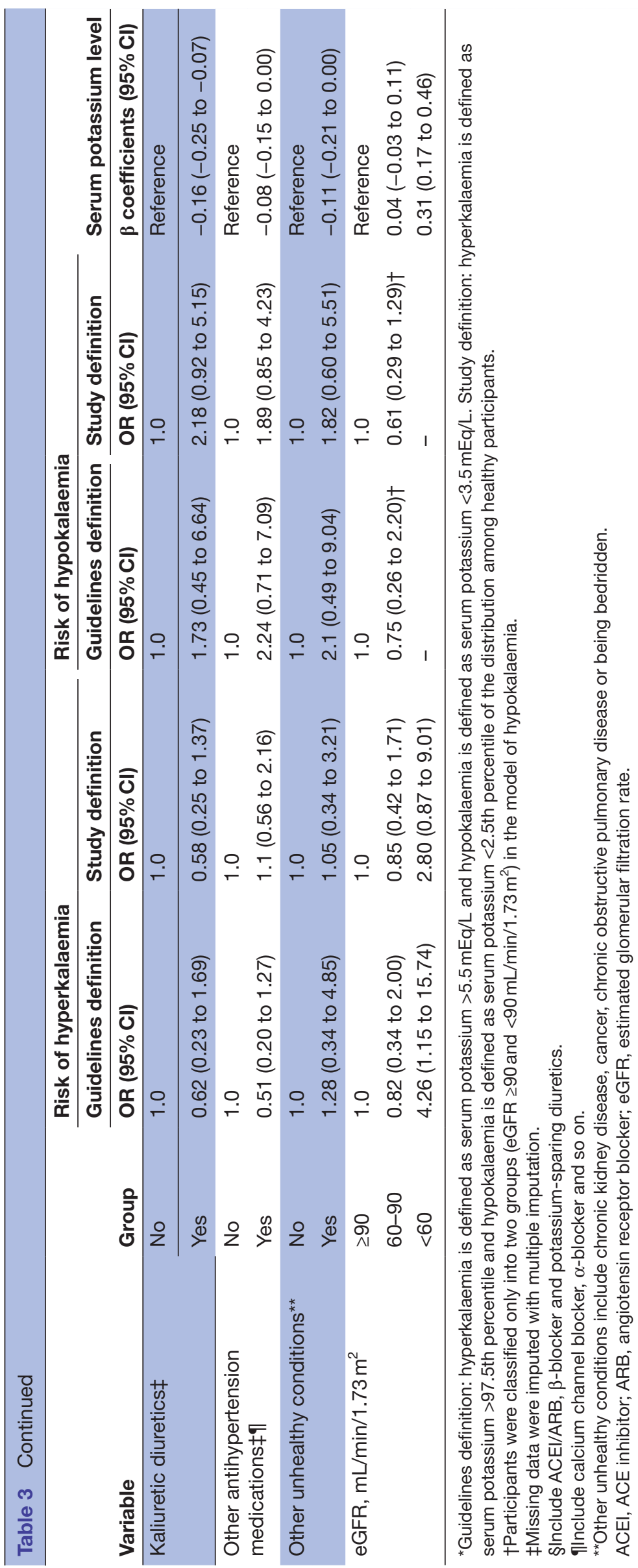


to the smaller number of participants on these medications and the complexity in the use of medications in real practice, where medication use differed very much from randomised trials.

Gender difference in mean serum potassium was also reported in the Renal Research Institute (RRI)-CKD study. ${ }^{31}$ The explanation that men have higher levels of serum potassium might simply be due to the higher amount of food intake among men and a rapid loss of potassium among women over 60 years. ${ }^{32}$

Geographical variations in serum potassium have also been previously reported in the literature..$^{33}$ Variations in serum potassium may reflect geographical variations in diet and seasonal changes in food availability. In fact, the baseline survey of the DECIDE-Salt trial was not done simultaneously across the four regions, starting with Changzhi in the fall of 2017 and ending with Yangcheng in the spring of 2018. Food variety and availability were usually highest in the fall and lowest in the spring in northern China.

\section{Strengths and limitations}

This is the first study on the epidemiology of dyskalaemia in a population living in elderly care facilities. The study facilities included assisted living homes, where a significant proportion of residents are healthy lonely persons in need of assisted living, which gave us the opportunity to explore the normal range of serum potassium of healthy Chinese older adults. To ensure their healthy status, we adopted a strict criteria that excluded any person with measured or reported major chronic diseases. Moreover, the facilities are regulated by the government and patients with any communicable diseases or any medical conditions that require hospitalisation are not allowed to be admitted. Considering previous studies were conducted in Western countries, the results from this study may shed light on Chinese and other Asian populations.

The study has several limitations. First, this is a crosssectional study and hence any inferences on causality should be interpreted cautiously. Second, our study participants were from elderly care facilities rather than from communities. The overall prevalence of hyperkalaemia and hypokalaemia in this study should not be extended directly to general older populations. Third, the subsample used to analyse the normal range of serum potassium should be considered as 'apparently' rather than 'entirely' healthy because our baseline examination included only basic physical and biomedical check-ups. However, we measured the known major factors that affect serum potassium homeostasis in every participant, and all those with these factors were excluded from the apparently healthy group. Fourth, the normal range of serum potassium could be better informed by data on the quantitative relationship of serum potassium level and its subsequent clinical outcomes. ${ }^{34} 35$ However, the present study was not able to provide these data. Fifth, over $80 \%$ of our participants were men. Due to Chinese culture, men are not accustomed to cooking meals in the family and hence are more likely to prefer living in elderly care facilities for assisted living when they have become widowers and have no other dependents. This led to the study population being predominantly men. Sixth, $21 \%$ of participants had not drawn blood sample and were not included in the study. They were older and predominantly women, with fewer smokers and drinkers, and with fewer patients with hypertension. Thus, the overall prevalence of hypokalaemia might be underestimated, while that of hyperkalaemia and mean serum potassium might be overestimated. Finally, the study sample may be not powered enough to draw conclusions on the negative findings of possible associated factors, such as potassium-sparing antihypertension medications and so on.

\section{CONCLUSION}

In conclusion, our study conducted among apparently healthy Chinese older adults suggests a normal range of serum potassium narrower than that used in current clinical guidelines. Our results also indicate that dyskalaemia is common among Chinese older adults. Unhealthy conditions would increase the risk of both hyperkalaemia and hypokalaemia, but the underlying mechanisms should be different. Future studies with larger sample size and cohort follow-up design will be required to confirm the causality of the associations found in the present study.

\section{Author affiliations}

${ }^{1}$ Peking University Clinical Research Institute, Peking University First Hospital, Beijing, China

${ }^{2}$ Renal Division, Department of Medicine, Peking University First Hospital, Beijing, China

${ }^{3}$ Department of Cardiology, China-Japan Friendship Hospital, Beijing, China ${ }^{4}$ Department of Preventive Medicine, Changzhi Medical College, Changzhi, Shanxi, China

${ }^{5}$ School of Public Health, Xian Jiaotong University, Xi'an, Shaanxi, China

${ }^{6}$ Yangcheng Ophthalmology Hospital, Jincheng, Shanxi, China

${ }^{7}$ Department of Nutrition and Food Safety, Hohhot Center for Disease Control and Prevention, Hohhot, Inner Mongolia, China

${ }^{8}$ Department of Epidemiology and Biostatistics, Peking University School of Public Health, Beijing, China

Acknowledgements We thank all study participants for their voluntary participation in the study and their resulting contribution to health science for the benefit of all. We also thank all investigators and staff of the participating nursing homes for their great support and wonderful cooperation in implementing the study.

Contributors YaW and AJ conceived and designed the study. AJ, XF, RZ, QQ, HW, JY, YuW, LC and HZ contributed to acquisition, analysis or interpretation of data. AJ conducted the data analysis. MZ, YS, H-JL and YaW provided administrative, technical or material support. AJ and YaW drafted the manuscript, which was critically reviewed and approved by all authors. YaW had full access to all of the data in the study and takes responsibility for the integrity of the data and the accuracy of the data analysis. YaW is the guarantor.

Funding This work was supported by the National Key Research and Development Program, Ministry of Science and Technology of China, through the research grant 'Diet, ExerClse and CarDiovascular hEalth, DECIDE project' (2016YFC1300200). AJ was supported by the China Scholarship Council (201806010389). The funding sources had no role in the design and conduct of the study; collection, management, analysis and interpretation of the data; preparation, review or approval of the manuscript; and decision to submit the manuscript for publication. 
Competing interests YaW received a small research grant to evaluate the efficacy and safety of a novel salt substitute manufactured by the Health Source (Chongqing) Cardiovascular Health Technology.

Patient consent for publication Not required.

Ethics approval The DECIDE-Salt trial was approved by the Peking University Institutional Review Board.

Provenance and peer review Not commissioned; externally peer reviewed.

Data availability statement Data are available upon reasonable request.

Supplemental material This content has been supplied by the author(s). It has not been vetted by BMJ Publishing Group Limited (BMJ) and may not have been peer-reviewed. Any opinions or recommendations discussed are solely those of the author(s) and are not endorsed by BMJ. BMJ disclaims all liability and responsibility arising from any reliance placed on the content. Where the content includes any translated material, BMJ does not warrant the accuracy and reliability of the translations (including but not limited to local regulations, clinical guidelines, terminology, drug names and drug dosages), and is not responsible for any error and/or omissions arising from translation and adaptation or otherwise.

Open access This is an open access article distributed in accordance with the Creative Commons Attribution Non Commercial (CC BY-NC 4.0) license, which permits others to distribute, remix, adapt, build upon this work non-commercially, and license their derivative works on different terms, provided the original work is properly cited, appropriate credit is given, any changes made indicated, and the use is non-commercial. See: http://creativecommons.org/licenses/by-nc/4.0/.

ORCID iD

Aoming Jin http://orcid.org/0000-0002-7221-5444

\section{REFERENCES}

1 Collins AJ, Pitt B, Reaven N, et al. Association of serum potassium with all-cause mortality in patients with and without heart failure, chronic kidney disease, and/or diabetes. Am J Nephrol 2017;46:213-21.

2 Coughtrie AL, Behr ER, Layton D, et al. Drugs and life-threatening ventricular arrhythmia risk: results from the Dare study cohort. BMJ Open 2017;7:e016627.

3 Palaka E, Grandy S, Darlington O, et al. Associations between serum potassium and adverse clinical outcomes: a systematic literature review. Int J Clin Pract 2020;74:e13421.

4 Schmidt M, Mansfield KE, Bhaskaran K, et al. Adherence to guidelines for creatinine and potassium monitoring and discontinuation following renin-angiotensin system blockade: a UK general practice-based cohort study. BMJ Open 2017;7:e012818.

5 Khow KSF, Lau SY, Li JY, et al. Diuretic-associated electrolyte disorders in the elderly: risk factors, impact, management and prevention. Curr Drug Saf 2014;9:2-15.

6 Fleet JL, Shariff SZ, Gandhi S, et al. Validity of the International classification of diseases 10th revision code for hyperkalaemia in elderly patients at presentation to an emergency department and at hospital admission. BMJ Open 2012;2:e002011.

7 Nilsson E, Gasparini A, Ärnlöv J, et al. Incidence and determinants of hyperkalemia and hypokalemia in a large healthcare system. Int J Cardiol 2017;245:277-84.

8 Chen Y, Chang AR, McAdams DeMarco MA, et al. Serum potassium, mortality, and kidney outcomes in the Atherosclerosis risk in Communities study. Mayo Clin Proc 2016;91:1403-12.

9 Liamis G, Rodenburg EM, Hofman A, et al. Electrolyte disorders in community subjects: prevalence and risk factors. Am J Med 2013;126:256-63.

10 Passare G, Viitanen M, Törring $\mathrm{O}$, et al. Sodium and potassium disturbances in the elderly. Clin Drug Investig 2004;24:535-44.

11 Kovesdy CP. Management of hyperkalemia: an update for the internist. Am J Med 2015;128:1281-7.

12 Khanna A, White WB. The management of hyperkalemia in patients with cardiovascular disease. Am J Med 2009;122:215-21.

13 Knochel JP. Diuretic-Induced hypokalemia. Am J Med 1984;77:18-27.
14 Jin A, Liu K, Labarthe DR, et al. Impact of salt substitute and stepwise reduction of salt supply on blood pressure in residents in senior residential facilities: design and rationale of the DECIDE-Salt trial. Am Heart J 2020;226:198-205.

15 Permitting Measures for the Management of Elderly Service Institutions. Order of the Ministry of Civil Affairs of the People's Republic of China No.48, 2013.

16 Wang Z, Chen Z, Zhang L, et al. Status of hypertension in China: results from the China hypertension survey, 2012-2015. Circulation 2018;137:2344-56.

17 Stamler J, Elliott P, Dennis B, et al. INTERMAP: background, aims, design, methods, and descriptive statistics (nondietary). J Hum Hypertens 2003;17:591-608.

18 McMurray JJV, Adamopoulos S, Anker SD, et al. Esc guidelines for the diagnosis and treatment of acute and chronic heart failure 2012: the task force for the diagnosis and treatment of acute and chronic heart failure 2012 of the European Society of cardiology. developed in collaboration with the heart failure association (HFA) of the ESC. Eur Heart J 2012;33:1787-847.

19 Yancy CW, Jessup M, Januzzi JL, et al. 2013 ACCF/AHA guideline for the management of heart failure: a report of the American College of cardiology Foundation/American heart association Task force on practice guidelines. Circulation 2013;128:e240-327.

20 Levey AS, Stevens LA, Schmid CH, et al. A new equation to estimate glomerular filtration rate. Ann Intern Med 2009;150:604-12.

21 Lee KJ, Carlin JB. Multiple imputation for missing data: fully conditional specification versus multivariate normal imputation. Am J Epidemiol 2010;171:624-32.

22 Jia K, Zhang C, Huang X, et al. Reference intervals of serum sodium, potassium, and chlorine in Chinese Han population and comparison of two ISE methods. J Clin Lab Anal 2015;29:226-34.

23 Kovesdy CP, Matsushita K, Sang Y, et al. Serum potassium and adverse outcomes across the range of kidney function: a CKD prognosis Consortium meta-analysis. Eur Heart J 2018;39:1535-42.

24 Acker CG, Johnson JP, Palevsky PM, et al. Hyperkalemia in hospitalized patients: causes, adequacy of treatment, and results of an attempt to improve physician compliance with published therapy guidelines. Arch Intern Med 1998;158:917-24.

25 Thomsen RW, Nicolaisen SK, Hasvold P, et al. Elevated potassium levels in patients with chronic kidney disease: occurrence, risk factors and clinical outcomes - a Danish population-based cohort study. Nephrology Dialysis Transplantation 2018;33:1610-20.

26 Jain N, Kotla S, Little BB, et al. Predictors of hyperkalemia and death in patients with cardiac and renal disease. Am J Cardiol 2012;109:1510-3.

27 Thomsen RW, Nicolaisen SK, Adelborg K, et al. Hyperkalaemia in people with diabetes: occurrence, risk factors and outcomes in a Danish population-based cohort study. Diabet Med 2018;35:1051-60.

28 Bayoumi E, Lam PH, Dooley DJ, et al. Spironolactone and outcomes in older patients with heart failure and reduced ejection fraction. Am J Med 2019;132:71-80.

29 Phillips CO, Kashani A, Ko DK, et al. Adverse effects of combination angiotensin II receptor blockers plus angiotensin-converting enzyme inhibitors for left ventricular dysfunction: a quantitative review of data from randomized clinical trials. Arch Intern Med 2007;167:1930-6.

30 Franse LV, Pahor M, Di Bari M, et al. Hypokalemia associated with diuretic use and cardiovascular events in the systolic hypertension in the elderly program. Hypertension 2000;35:1025-30.

31 Korgaonkar S, Tilea A, Gillespie BW, et al. Serum potassium and outcomes in CKD: insights from the RRI-CKD cohort study. Clin J Am Soc Nephrol 2010;5:762-9.

32 Flynn MA, Nolph GB, Baker AS, et al. Total body potassium in aging humans: a longitudinal study. Am J Clin Nutr 1989;50:713-7.

33 Beusekamp JC, Tromp J, van der Wal HH, et al. Potassium and the use of renin-angiotensin-aldosterone system inhibitors in heart failure with reduced ejection fraction: data from BIOSTAT-CHF. Eur $J$ Heart Fail 2018;20:923-30.

34 Thongprayoon C, Cheungpasitporn W, Thirunavukkarasu S, et al. Serum potassium levels at hospital discharge and one-year mortality among hospitalized patients. Medicina 2020;56:236.

35 Thongprayoon C, Cheungpasitporn W, Hansrivijit P, et al. Admission serum potassium levels in hospitalized patients and one-year mortality. Medicines 2019;7:medicines7010002:2. 